\title{
Experimental estimation of GNSS performances at the national aviation university
}

\author{
Volodymir Kharchenko ${ }^{1, *}$, Valeriy Konin ${ }^{1}$, Olexiy Pogurelsky ${ }^{1}$ and Ekaterina Stativa $^{2}$ \\ ${ }^{1}$ National Aviation University, Kyiv, Ukraine \\ ${ }^{2}$ Moscow State University of Civil Engineering, 26 Yaroslavskoye Shosse, Moscow, 109377, Russia
}

\begin{abstract}
The goal of the research is to develop a of Global Navigation Satellite Systems quality monitoring methodology based on available equipment in the satellite navigation laboratory of the National Aviation University (Kyiv, Ukraine). For successful the goal achievement it is necessary to solve follow list of tasks: to determine the composition of the necessary equipment and order of it installing and connection; to develop the necessary software for processing received GNSS data; to estimate the GNSS characteristics with the help of experimental data. The primary focus of this research is on the following characteristics: accuracy (in terms of deviation coordinates in horizontal and vertical planes from the coordinates of the reference station and numerical values in meters); integrity information (summarized in the form of horizontal and Stanford plots); overall availability of service - measured as the availability of signals meeting the requirements for instrumented approaches with vertical guidance (APV) APV-1, APV-2, and Category 1 (CAT-1) precision approaches to runways. The main result of this research is developing software that could be applied for continuous monitoring of GNSS performances. The possibilities of it were successfully tested with the help of experimental data received from GPS and Galileo satellites.
\end{abstract}

\section{Introduction}

Technologies of Global Navigation Satellite Systems (GNSS) are widely used today in the transportation industries. It could be used for monitoring ground traffic, creating efficient agricultural solutions, free and accurate mapping, various maritime uses, and other location-based services. The quality of GNSS services could be estimated with the help of accuracy, integrity and availability.

Accuracy is the degree of conformance between the measured or estimated position and the true position of an aircraft at a given time.

Integrity is a measure of the trust which can be placed in the correctness of the information supplied by the total system. Integrity includes the ability of the system to alert

\footnotetext{
* Corresponding author:kharch@nau.edu.ua
} 
the user when the system should not be used for the intended operation (or phase of flight). The necessary level of integrity for each operation is established with respect to specific horizontal/lateral (and for some approaches, vertical) alert limits.

The goal of the research is to develop a GNSS quality monitoring methodology based on available equipment in the satellite navigation laboratory of the National Aviation University (Kyiv, Ukraine). For successful the goal achievement it is necessary to solve follow list of tasks:

1. To determine the composition of the necessary equipment and order of it installing and connection;

2. To develop the necessary software for processing received GNSS data;

3. To estimate the GNSS characteristics with the help of experimental data. The primary focus of our research will be on the following characteristics:

- accuracy (in terms of deviation coordinates in horizontal and vertical planes from the coordinates of the reference station and numerical values in meters);

- integrity information (summarized in the form of horizontal and Stanford plots);

- overall availability of service - measured as the availability of signals meeting the requirements for instrumented approaches with vertical guidance (APV) APV-1, APV-2, and Category 1 (CAT-1) precision approaches to runways [1-14].

\section{Equipment description}

Fig. 1 shows a block diagram of data flow and main equipment that should be used for it processing.

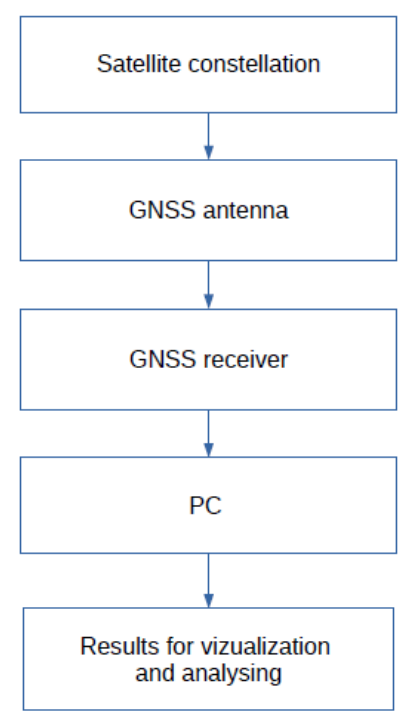

Fig. 1. Block diagram of data flow and main equipment.

The satellite constellation consist of all space vehicles different navigation systems that are functioning on Earth orbits and perform the functions of broadcasting special navigation messages. All of these satellites could be applied as sources of information that users need for performing navigational calculations. It should be provided possibility to receive data from all available satellite navigation systems (GPS, Galileo, GLONASS, Beidou) and their combinations 
The receiving GNSS antenna is located on the roof of the eleventh building of National Aviation University (fig. 2).

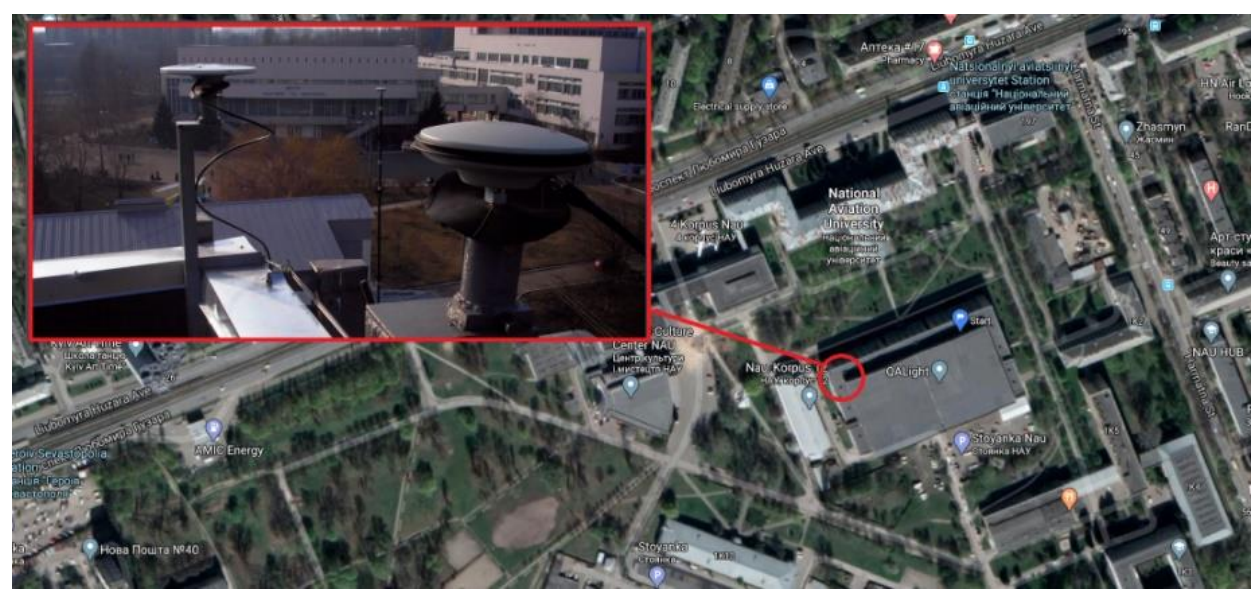

Fig. 2. The location of GNSS antenna.

Fig. 3 shows a compact antenna located inside the laboratory. It is used for receiving of satellite signals indoors. It is advisable to use antennas mounted on the roof of the building to investigate the parameters of a full-fledged navigation field with the possibility of receiving a signal from the maximum number of satellites. The connection to the receivers inside the laboratory is realized through cables that are brought into the laboratory, as shown in fig. 4 and further distributed into several receiving sets by means of splitters with active signal amplification.

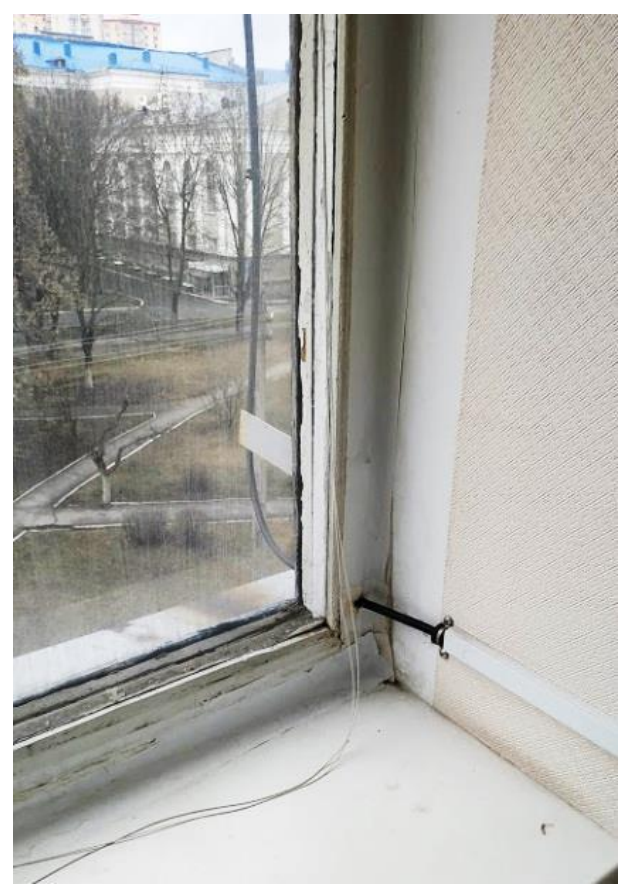

Fig. 3. Compact antenna located inside the laboratory. 


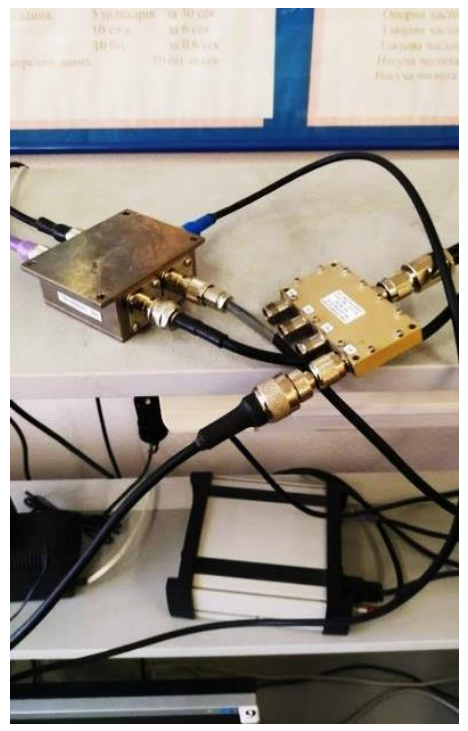

Fig. 4. Connection to the receivers inside the laboratory.

Satellite receiver is the key element of developed monitoring complex. Note that to perform the set of tasks required receivers of a special class, with the ability to access the "raw" navigation messages, measurement results and a number of other official information. These requirements are satisfied by the receivers manufactured by Novatel, shown in fig. 5 and fig. 6.

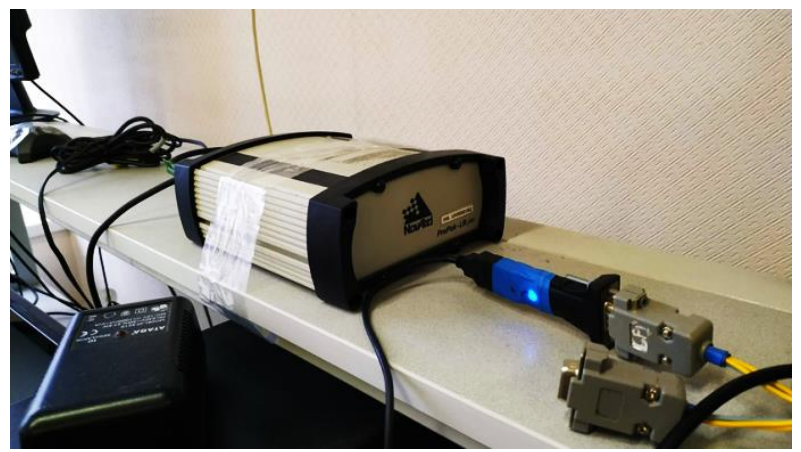

Fig. 5. Receivers manufactured by Novatel.

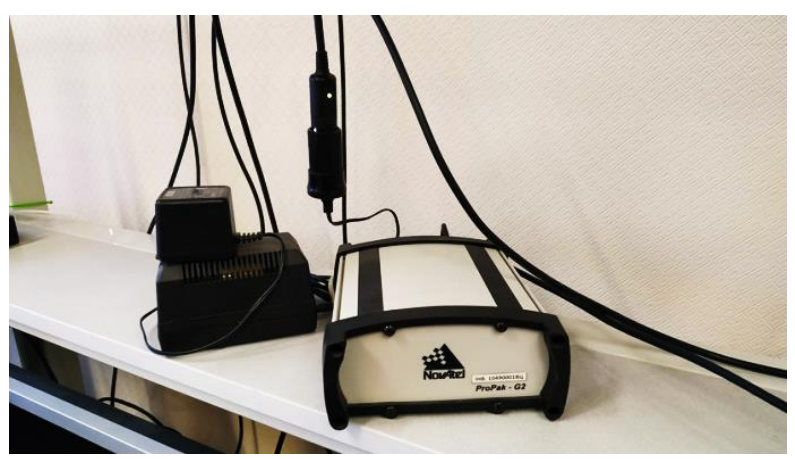

Fig. 6. Receivers manufactured by Novatel. 
Processing, storing, visualization and analyzing data is performed in a workplace equipped with a personal computer (PC). Fig. 7 shows PC with installed software for communication with receiver - receiver interface. It allows user to generate requests for data to be received with the receiving equipment. The Novatel Connect interface is a fairly convenient and widespread software for sharing data with the Trimble family of receivers. Depending on the version, its appearance may vary, but it must contain the feature of the data set (the so-called log file), which must be obtained from the antenna system from the navigation satellites. It should be noted that this log file may also include data obtained by the calculation resulting from the secondary processing performed inside the receiver.

\section{Software facilities}

The experimental research consists of receiving GNSS data and integrity information, generated by receiver as a result of Receiver Autonomous Integrity Monitoring Algorithm (RAIM) functioning. This information gives the capability of calculating a confidence interval, alerting them when a GNSS malfunctions and is not to be used for safety-lifeapplication.

The alert limit (AL) is a fixed threshold corresponding to a type of operation.

The mechanism to trigger an integrity alert compares, for each epoch, a (conservative) estimate of the position accuracy in relation to the alert limit. This estimate, called the protection level (PL), is computed based on quality estimates provided by tropospheric, ionospheric, and SARPS variance models embedded in the receiver software. The PL provides an indication of error uncertainty modeled by the variance of a zero-mean normal distribution that describes user differential range errors, user ionospheric range error, aircraft pseudorange errors due to multipath, and residual pseudorange errors from a tropospheric model.

An integrity event is an epoch in which the position error (PE) exceeds a maximum allowable alert limit, while no alert is generated within an allowable time period, called the Time to Alert (TTA).

A misleading information (MI) event is considered as every epoch in which PE is greater than the PL, which can be regarded as reflecting a system anomaly. Hazardously misleading information (HMI) is defined as every epoch where the position error is greater than the alert limit and the protection level, which represents an anomaly and can be hazardous for users. HMI thus means that the epoch is actually unavailable but would have been labeled as a valid one in a flight. (Note that ALs can differ for various types of users/operations.) In HMI situations the actual position error is greater than the alert limit $(\mathrm{PE}>\mathrm{AL})$ and the alert limit is larger than the protection level $(\mathrm{AL}>\mathrm{PL})$ but the epoch still passed as valid for this operation because the protection level was estimated incorrectly.

The interface of the navigation receiver allows you to record a wide list of different types of data that come both directly from GNSS navigation satellites and are formed inside the receiver as a result of operating in the established mode.

The location of the receiver at a point with known coordinates can be used to find a positioning error, as the difference between the estimation of coordinates according to GNSS and known coordinates. A message that allows you to accumulate an array of coordinate estimates is a message type 42 BESTPOS. The information in the BESTPOS message contains data about the user's coordinates, which are calculated from the data obtained from available navigation satellites in the form of longitude, latitude and altitude.

Messages of type 1186 RAIMSTATUS were required to evaluate the integrity. It provides an assessment of the integrity parameter - the horizontal and vertical levels of HPL and VPL. 
Recorded log files contain information in a format, so a further decoding process takes place in a program created in MatLab.

MatLab is a convenient tool for working with algorithms as well as for visual analysis of data, which is implemented in a large number of functions for plotting graphs, including three-dimensional ones.

The simplified algorithm of operation of the program is given below:

1. Opening a log file;

2. Reading the message ID number;

3. The message is decoded according to the received number: if the number is 1286 , then the RAIM message decoding function opens, and if 42 , the BESTPOSA decoding function.

4. The decoding process gives latitude, longitude and altitude coordinates in degrees, their deviations, time values, and for the RAIM message also HPL and VPL. All data is stored in separate vectors to facilitate their use in graphing.

5. After processing the messages, determine the true location of the receiver by averaging all the values of latitude, longitude and height.

6. Coordinates from the geodetic are transformed into the ECEF coordinate system.

7. The values of the exit threshold beyond the permissible deviation are further specified: for precise approach $-12 \mathrm{~m}$, for approach with vertical control $-20 \mathrm{~m}$, and in the horizontal plane $-40 \mathrm{~m}$.

8. Stanford Plot visualization based on decoded and processed data.

\section{Research results}

In this article, we will use the results of research conducted on December 2019, to provide an example of our experimental program's findings.

Set of received data was obtained for GNSS constellation with 9 GPS satellites and 8 Galileo. The geometry of their visibility is shown on fig. 7 .

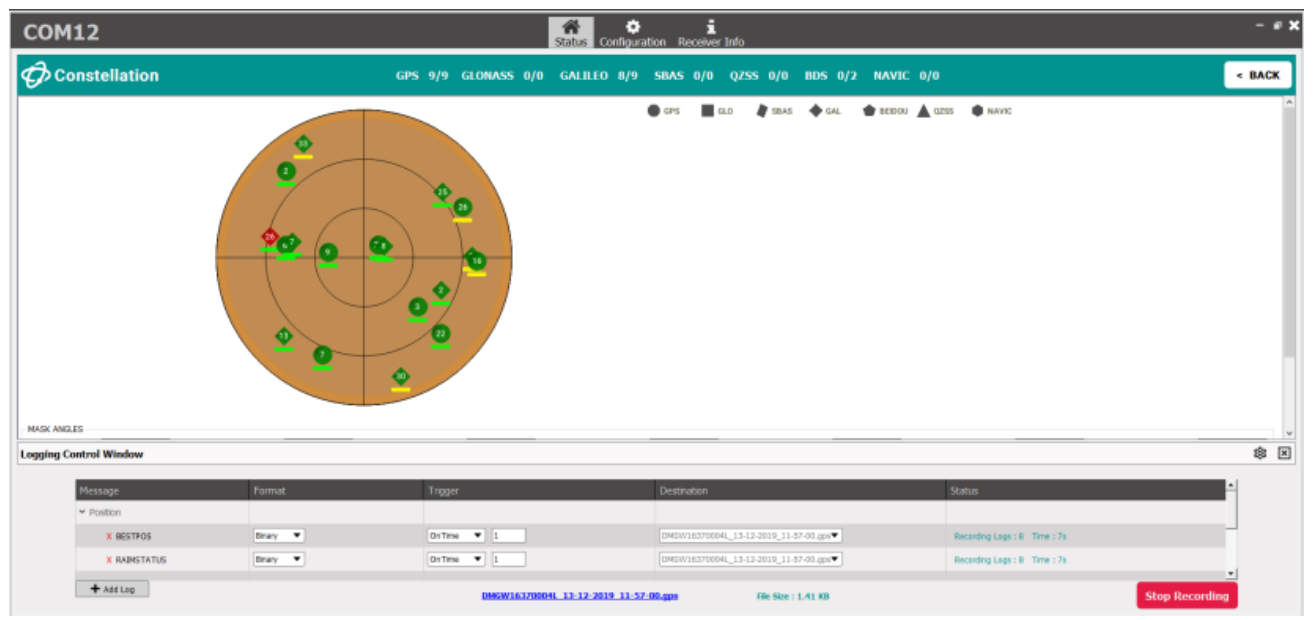

Fig. 7. The geometry of visibility of the satellites.

Results of processing received data are shown on fig. 8-12.

Positioning error in horizontal plane (HPE) and horizontal protected level (HPL) during period of recording/monitoring: 


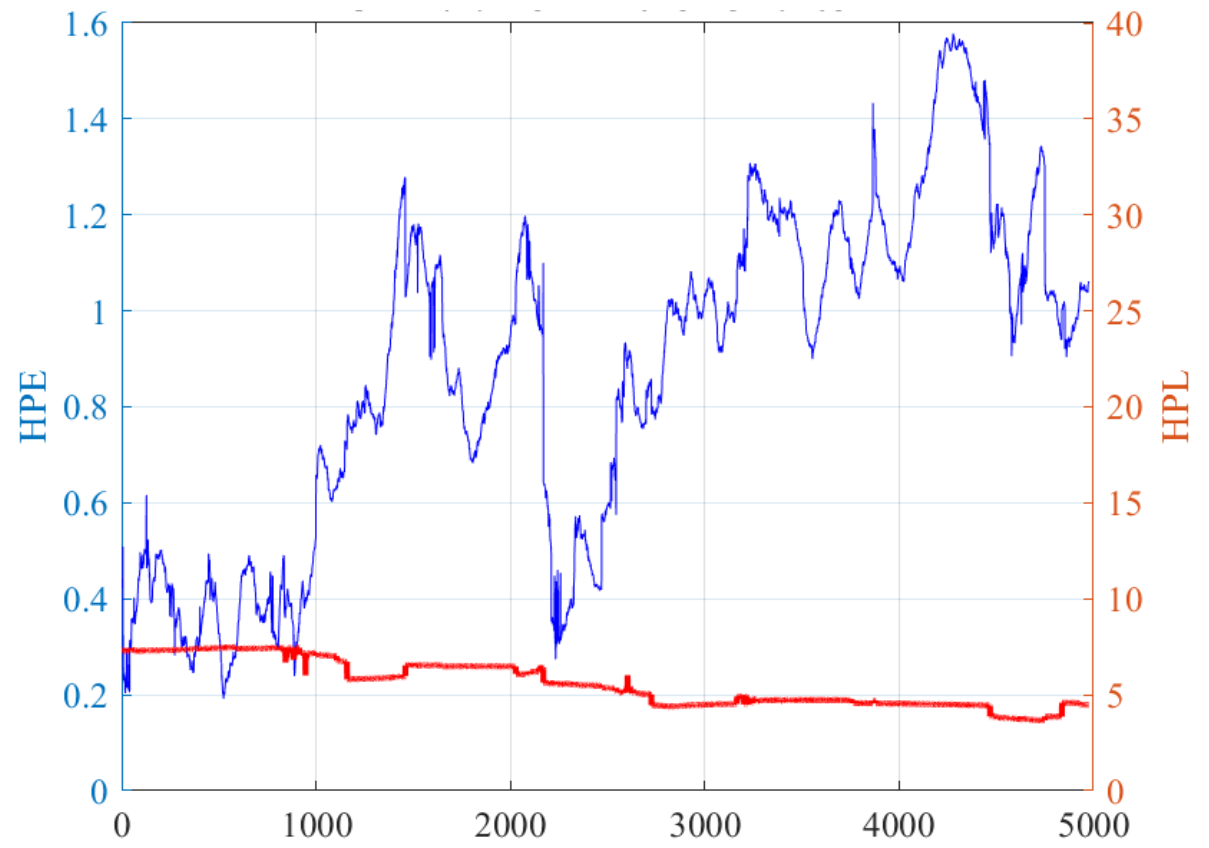

Fig. 8. Results of processing received data.

Positioning error in vertical plane (VPE) and vertical protected level (VPL) during period of recording/monitoring:

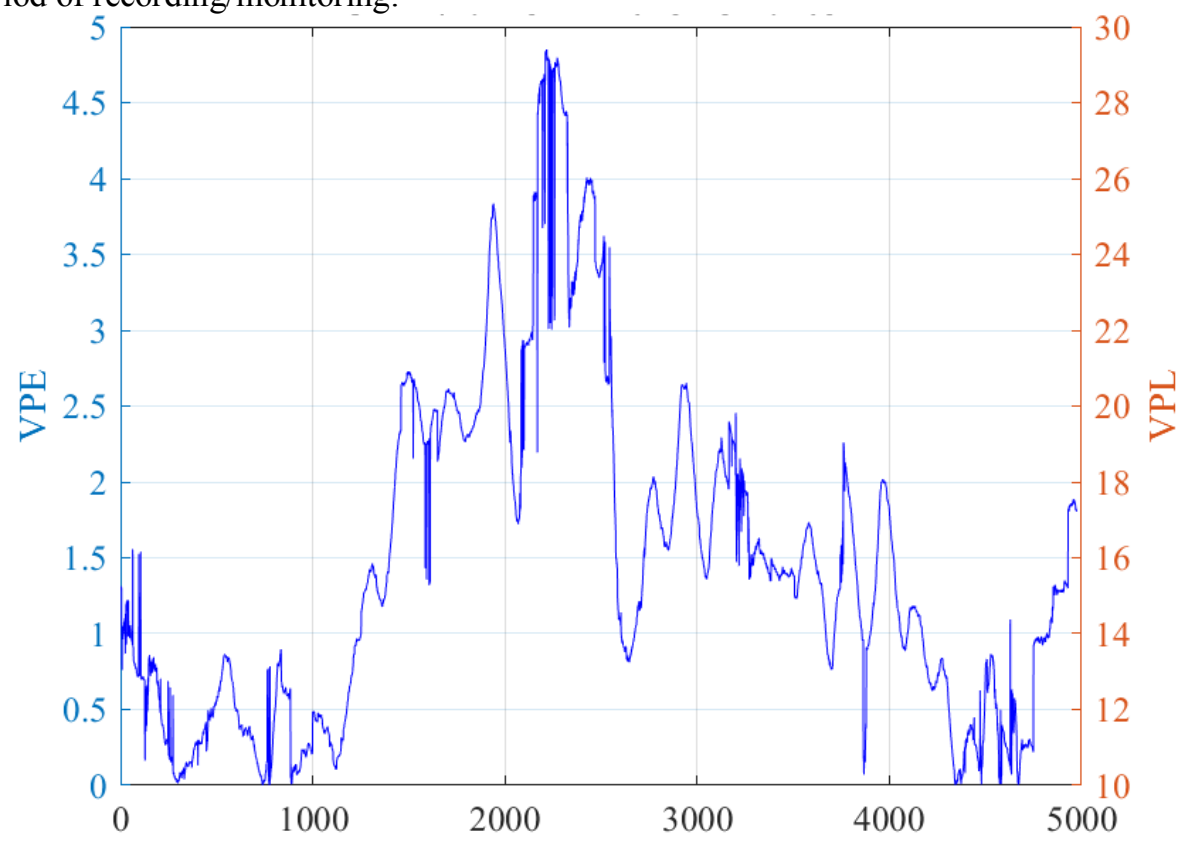

Fig. 9. Results of processing received data.

The accuracy achieved in the horizontal plane displayed as north-south and east-west deviations from the primary reference point. 


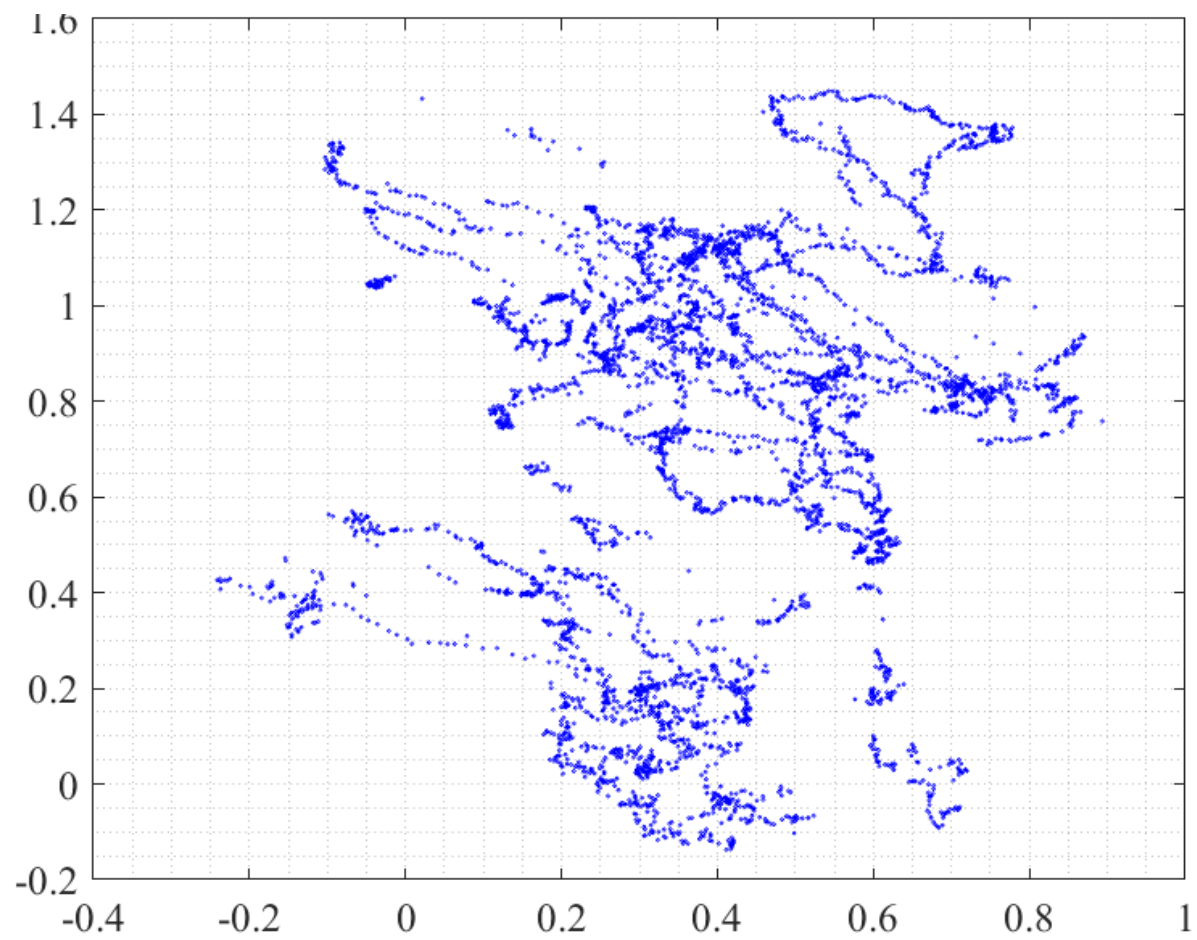

Fig. 10. Results of processing received data.

Horizontal performance of GNSS (combination GPS+Galileo) during monitoring period on December 13, 2019.

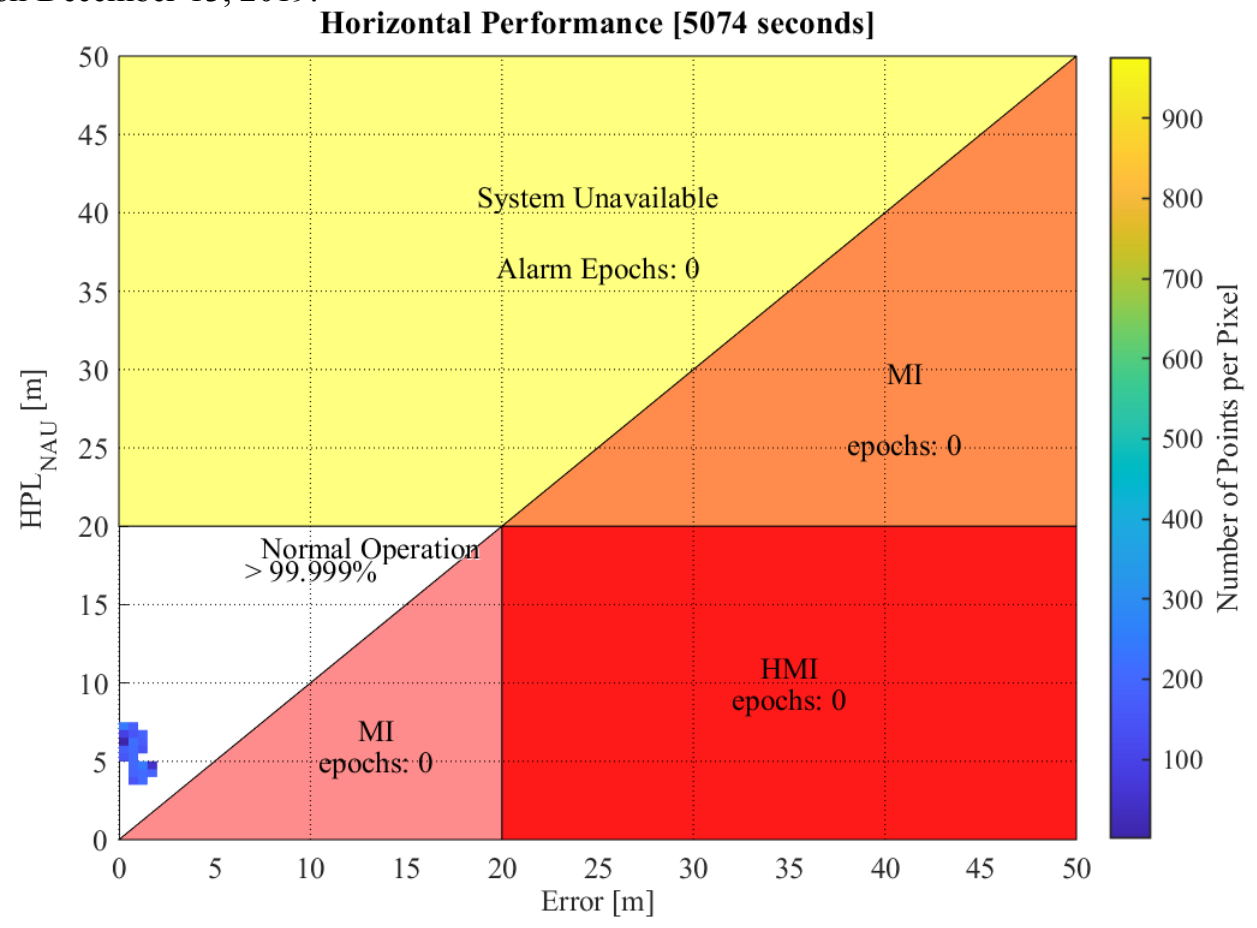

Fig. 11. Results of processing received data. 
Vertical performance of GNSS (combination GPS+Galileo) during monitoring period on December 13, 2019.

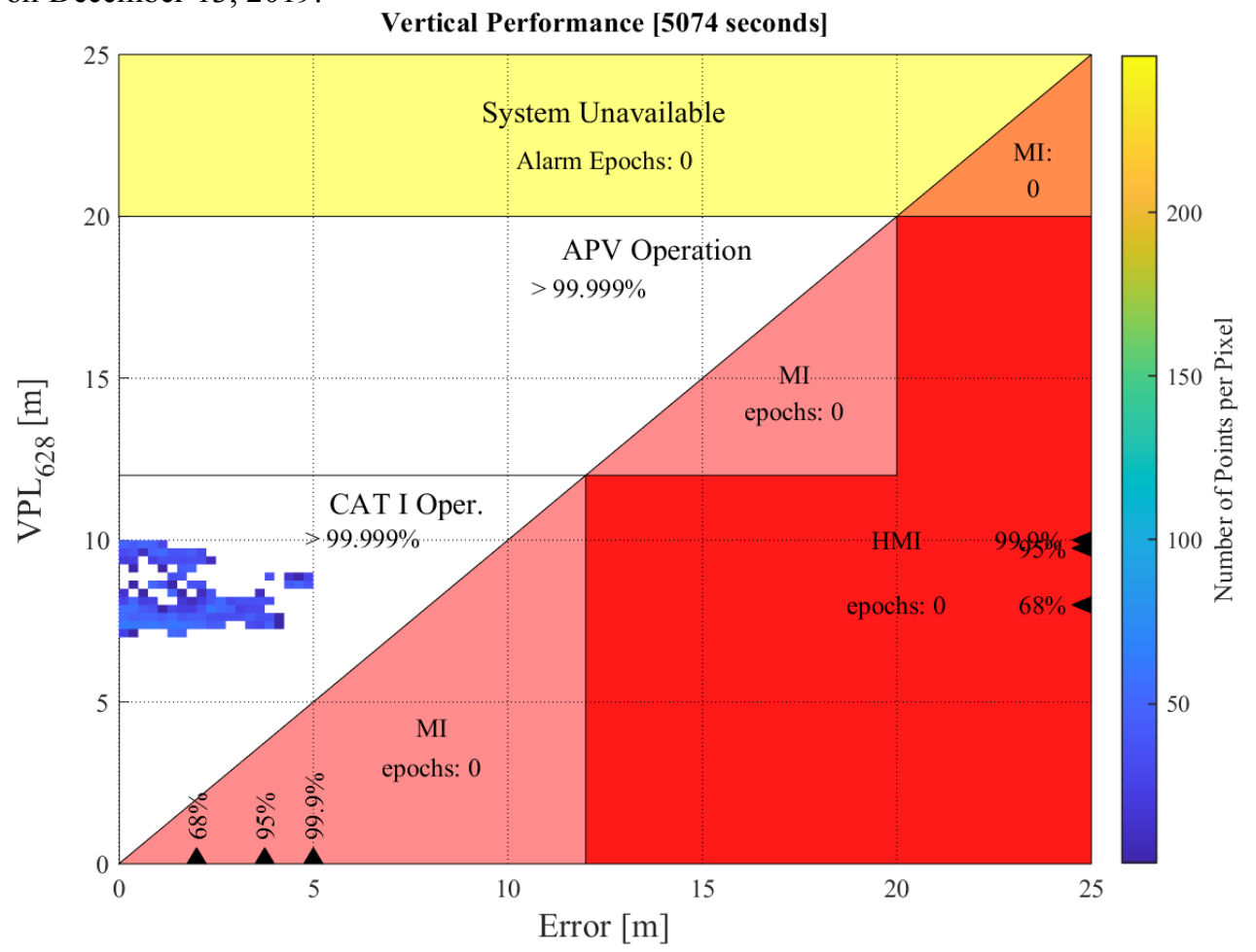

Fig. 12. Results of processing received data.

To summarize the integrity information generated by our research, we used the format developed by Stanford University to characterize performance of the U.S. Wide Area Augmentation System (WAAS). The measurements that correspond to typical operations of APV-1, APV-2, and CAT-1 for horizontal and vertical planes are shown on fig. 11 and fig. 12. On the horizontal axes, precision errors are plotted for horizontal (HPE) and vertical (VPE) planes; on the vertical axes we have alarm limits for horizontal (HPL) and vertical planes (VPL), respectfully.

The color scale allow us to calculate the number of points (therefore the number of epochs) that meet - or, conversely, fail to meet - the required performance for various approach and landing procedures. All epochs that meet a stricter standard will satisfy a lower standard.

For 5074 valid epochs in the horizontal and vertical plane, all of them were fit for safety-critical operations and satisfied CAT-1. Such figures can only be built on the ground, as we have no position errors information during the flight.

\section{Conclusions}

The main result of this research is developing software that could be applied for continuous monitoring of GNSS performances. The possibilities of it were successfully tested with the help of experimental data received from GPS and Galileo satellites.

There were no integrity concerns and therefore we may conclude that GNSS combination from GPS and Galileo satellite constellations is safe to use from the integrity point. 


\section{References}

1. Global Positioning System Wide Area Augmentation System (WAAS) Performance Standard, 1st edition (FAA, 2008)

2. Aeronautical Telecommunications Annex 10 to the Convention on International Civil Aviation 1, (International Civil Aviation Organization, 2001)

3. V. Konin, F. Shyshkov, InsideGNSS, 50-54 (2015)

4. L. Lazarević, D. Vučković, Z. Popović, Journal of Rail and Rapid Transit 230(8), 1828-1841 (2016).

5. M. Vilotijević, M. Vukićević, L. Lazarević, Z. Popović,Tehnički vjesnik 25(2), 516523 (2018).

6. L. Lazarević, D. Vučković, M. Vilotijević, Z. Popović, Structural Health Monitoring 18(3), 792-805 (2019).

7. V. Vorobyov, A. Manakov, I. Yanshina, I. Repina, Advances in Intelligent Systems and Computing, VIII International Scientific Siberian Transport Forum 2, 1116 (2020). DOI: 10.1007/978-3-030-37919-3_69

8. T. Vladimirova, A. Manakov, V. Sokolov, MATEC Web of Conferences 216, 02008 (2018). DOI: $10.1051 /$ matecconf $/ 201821602008$

9. V. Vorobyov, A. Manakov, A. Reger, I. Tanaino, MATEC Web of Conferences 216, 02009 (2018). DOI: 10.1051/matecconf/201821602009

10. Ilinykh, A. Manakov, A. Abramov, S. Kolarzh, MATEC Web of Conferences 216, 03004 (2018). DOI: 10.1051/matecconf/201821603004

11. A.L. Manakov, A.D. Abramov, A.S. Ilinykh, M.S. Galay, J.S. Sidorov, Journal of Physics: Conference Series 1050(1), 012051 (2018). DOI: 10.1088/17426596/1050/1/012051

12. K. Kornienko, S. Bessonenko, I. Tanaino, Advances in Intelligent Systems and Computing 1115, 655-662 (2020). DOI: 10.1007/978-3-030-37916-2_63

13. Tanaino, O. Yugrina, L. Zharikova, IOP Conference Series: Earth and Environmental Science 403(1), 012192 (2019). DOI: 10.1088/1755-1315/403/1/012192

14. S. Bessonenko, K. Kornienko, I. Tanaino, MATEC Web of Conferences 239, 03002 (2018). DOI: $10.1051 /$ matecconf/201823903002 